\title{
Structural response of reduced scale naval structures under impact tests
}

\author{
M.A.G. Calle, R.E. Oshiro, L.M. Mazzariol and M. Alves \\ Department of Mechatronics and Mechanical Systems, Polytechnic School of the University of Sao Paulo, \\ Sao Paulo, SP, 05508-900, Brazil
}

\begin{abstract}
Scaled models are important in naval engineering since actual ship size makes too expensive to test prototypes. However, the analysis of ship collision events employing naval structures in reduced scale is not an ordinary ship research area. The aim of this work is to create the basis for a posterior similarity study by analysing reduced scale ship structures submitted to impact loads. Two basic naval structures, commonly found in the construction of large ships, were considered for this study: a T cross-section beam submitted to a midspan impact test and a double plate panel with inner cross reinforcement also submitted to a central impact load. These models were made in a reduced scale of 1:100. The experimental material characterization was also carried out in this work, including the evaluation of the stress strain curve under quasi static conditions, the strain rate sensitivity and the structural failure using three criteria developed particularly for numerical modelling of ship collision by other authors.
\end{abstract}

\section{Introduction}

The majority of the most catastrophic accidents of ships occurs due to collision and grounding [1]. These accidents are associated with areas of intense ship traffic and offshore operations such as oil production rigs. Numerous accidents are caused by human error or failure of the ship, but also by harsh environment conditions. The risk of ship collision had increased together with the growth of the global fleet of ships. Oil tankers represent about $48 \%$ of the world fleet and they are the maritime segment in which the largest ships are built [1]. These large size vessels are very efficient in transporting oil, but risk a great amount of oil leakage in a possible collision event. The injury caused by a ship collision accident not only causes oil spill and ship structure damage but also can cause degradation of the marine environment, explosions, human losses, blocking of ships traffic and permanent damage to the ship. This global scenario emphasizes the necessity of reformulating the safety in the entire offshore oil exploration chain, as well as investing in better ship designs.

Models in reduced scale are important in naval engineering since the experimental test of real-size ships can be too difficult and expensive. However, only hydrodynamic ship aspects are traditionally evaluated by scaled models. The study of the structural behaviour of ships during a ship collision event using reduced scale model is not a common study in the ship research community [2]. The large quantity of structural details of a ship structure is one of the principal difficulties to overcome when working in a reduced scale; some aspects such as non-similarity in material failure and omission of structural member to simplify a scaled construction have significant influence in the results [3].

Other of the critical challenges in the finite element modelling of the collision of thin plate structures is the developing of an adequate mechanical characterization of the structural material $[2,4,5]$. During an impact event of a thin plate structure, both force-displacement response and structural collapse mode of the collided structure are strongly dependent on material parameters as the true stress strain curve, strain rate sensitivity and failure criterion as well as geometric imperfections induced in the manufacturing process as welding distortions and quality, assembly misalignments, among others.

\section{Material model}

\subsection{Elastic plastic behaviour}

The reduced scale naval structures were fabricated using mild steel plates (SAE 1008) due to its similar mechanical properties to naval steels. The plate was conceived to have $0.25 \mathrm{~mm}$ thickness to better represent welded naval structures in a reduced scale of $1: 100$.

The limitations to obtain the plastic behaviour of a material using standard uniaxial tensile tests begin when the necking initiates because the strain distribution in the section of the specimen is no longer uniform and the strain history will not represent the real local plastic behaviour. Even using the displacement of the machine 
crosshead, a clip gage or two points tracking at any relative distance, the estimated curve will stop following the real stress strain curve of the material. By using photogrammetric techniques, it is possible to widen the extent of the stress strain curve acquisition to values close to twice the strain range of traditional methods. However, these techniques are limited to the number of images acquired, minimum element size and the maximum supported distortion of the pattern painted in the specimen surface. On the other hand, the optimization procedure allows to obtain the entire stress strain curve until complete failure, thanks to fine tuning of the material parameters [6].

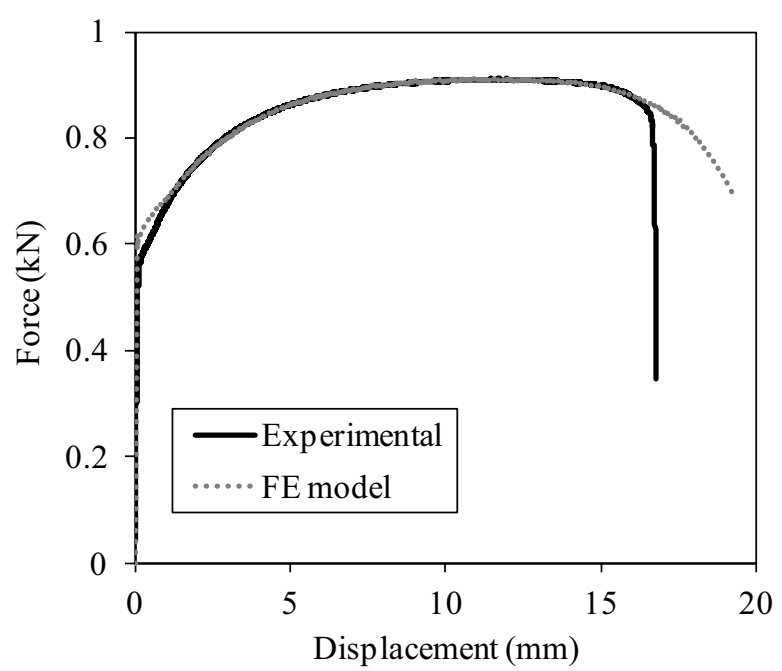

Fig. 1. Experimental and numerical force displacement curves of tensile test in the optimization routine.

So an optimization procedure was performed to obtain the material parameters of mild steel plate, with the result depicted in Fig. 1. This procedure was based in the adjustment of the force displacement response of the numerical model to the analogous experimental response in a uniaxial tensile test via the fine tuning of the material model parameters to attain the desirable response [6]. The experimental force displacement response was acquired by tensile tests executed in an Instron universal testing machine, model 3369. The specimen dimensions were $50 \times 10 \times 0.25 \mathrm{~mm}$. The optimization routine was made in Pascal language and the LS-dyna code was used in the FE model processing in explicit analysis. The $2 \mathrm{D}$ model was created using square $0.5 \times 0.5 \mathrm{~mm}$ shell elements.

The Hughes-Liu formulation [7] and five integration points through the thickness were considered for the shell elements. The Voce material model was implemented in the LS-dyna due to the better description of the plastic behaviour of the material by using four material parameters, equation (1).

$\sigma=k+R_{0} \cdot \varepsilon+R_{\infty}\left(1-e^{-b \varepsilon}\right)$

where $k$ is the yield stress, obtained directly from the experimental test, and the others three variables $\left(R_{0}, R_{\infty}\right.$ and $b$ ) needs to be adjusted in the optimization routine. The optimization loop ends when difference between experimental and numerical force displacement curves attained a minimum value for a determined tolerance in Voce parameters which resulted in $k=219.0 \mathrm{MPa}, R_{0}=$ $479.8220 \mathrm{MPa}, R_{\infty}=126.2866 \mathrm{MPa}$ and $b=21.4768$.

\subsection{Dynamic sensitivity}

In general, carbon steel is very sensitive to high strain rates, exhibiting a higher material resistance as straining velocity increases. Due to the dynamic nature of collision events, the dynamic sensitivity of the material needed to be introduced in the material model by using the CowperSymonds model. The dynamic increment factor $(D I F)$, defined as the true stress obtained at dynamic test conditions divided by the true stress under quasi static test conditions, can be estimated by the Cowper-Symonds sensitivity model [8] in which $C$ and $p$ are parameters of the material, equation (2).

$D I F=1+\left(\frac{\dot{\varepsilon}}{C}\right)^{1 / p}$

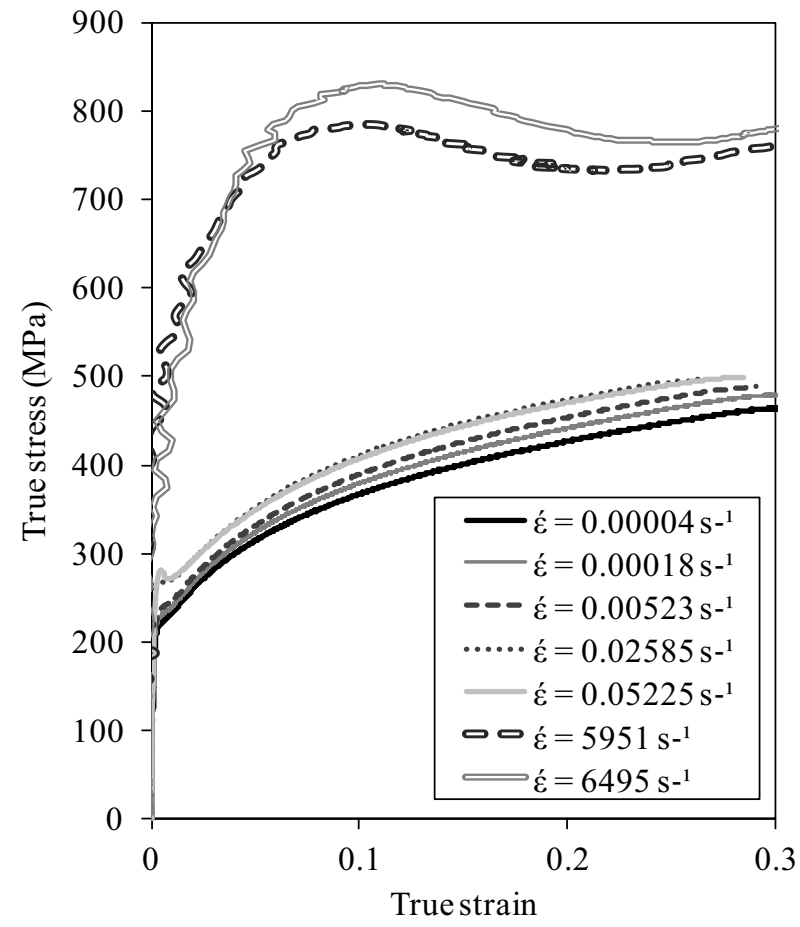

Fig. 2. True stress strain curves obtained from experimental tests at low and high velocities.

A series of tests were performed to evaluate the sensitivity of the true stress strain curves to the strain rates characterized by the Cowper-Symonds parameters. These tests were divided into two groups: low and high velocity tests. For the low velocity tests, uniaxial tensile tests were performed in an Instron universal testing machine, model 3369, achieving test velocities from 0.1 to $150 \mathrm{~mm} / \mathrm{min}$, yielding strain rates in the range of 0.00004 to $0.05 \mathrm{~s}^{-1}$. For the high velocity tests, disc specimens were tested in a split Hopkinson pressure bar 
with strain rates about $6000 \mathrm{~s}^{-1}$. Figure 2 shows the true stress strain curves for all the tests, each one characterized by the respective strain rate.

Using a logarithmic approach and evaluating the true stress for all curves at 0.2 total strain, figure 2 , the Cowper-Symonds parameters can be obtained using a linear fitting of the DIF and the average strain rate of the tests. The true stress strain obtained from the tensile test performed at the lowest test velocity $(0.1 \mathrm{~mm} / \mathrm{min})$ was considered as the reference curve. This analysis resulted in $C=24422.58 \mathrm{~s}^{-1}$ and $p=4.6889$ and the DIF prediction as shown in figure 3 .

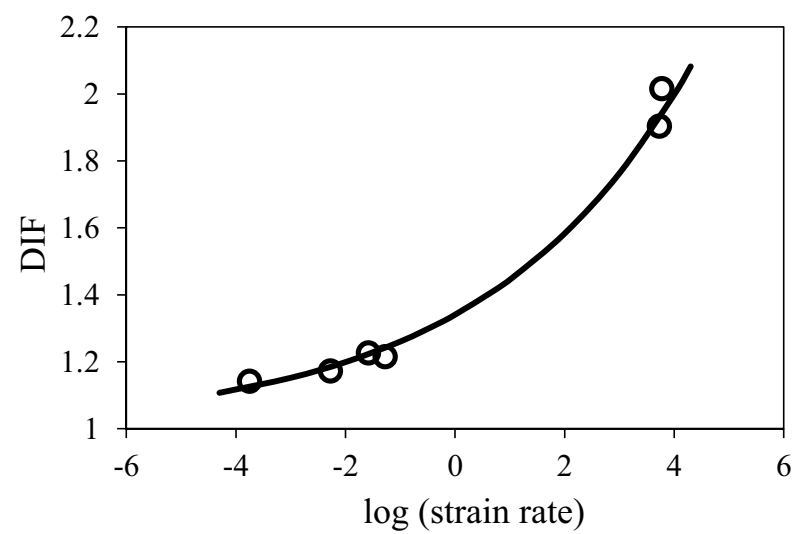

Fig. 3. Prediction of the dynamic increment factor using the Cowper-Symonds model.

These parameters were introduced in the numerical model for the optimization procedure to evaluate the Voce material model parameters so aiming to include the dynamic sensitivity in the material.

\subsection{Failure criteria}

On numerical simulation of ship collision it is very important that the failure criterion of the structure would be correctly established because of its strong influence on the global structural energy absorption and the fracture extension in structures [1]. In fact, the assessment of the structural failure in a ship collision event is a very complex process influenced by many factors as the mechanical properties of the structural materials, geometry of the structural members, constructive imperfections, application of the loads, velocity of the loads as many others. These diverse factors can promote different collapse modes normally observed in collided naval structures as cutting, concertina tearing, folding, crushing, ruptured plate and membrane stretching [9]. To reproduce a similar structural response by numerical modelling of a ship collision, an adequate failure criterion should promote most of these collapse modes at larger scales. It can also be applied in reduced scale naval structures where the failure criterion needs to reproduce joints detaching and plate ruptures.

Three failure criteria were used in this work: the equivalent plastic strain (EPS) criterion, the Rice-TraceyCrockroft-Latham (RTCL) criterion and the BressanWilliams-Hill (BWH) criterion. The material parameters for the failure criteria models were obtained from the evaluation of the numerical modelling of the tensile test experiment.

The EPS criterion uses a maximum equivalent plastic strain value associated with the material failure, equation (3). This criterion was commonly used in ship to ship collision modeling $[10,11]$ to described energy absorption due to structural collapse, given that the maximum plastic strain is dependent on the mesh size, load application and structural part localization. The failure parameter for the $0.5 \times 0.5 \mathrm{~mm}$ mesh size was evaluated in 0.93648 .

$D=\int_{0}^{\bar{\varepsilon}} f d \bar{\varepsilon}$

The RTCL criterion was based in the combination of two well-known failure models, the Rice-Tracey and the Crockcroft-Latham criteria to cover the full triaxiality range satisfactorily [12], equation (4), Crockroft-Latham for ductile shear fracture (low triaxialities range) and the Rice-Tracey for void growth (high triaxialities range) $[13,14]$. The Crockroft-Latham criterion was expressed entirely as a function of the triaxiality value [15], equation (5). The RTCL criterion was validated by numerical modelling of several large-scale experiments for various ship accident scenarios including indentations and tearing of the ship bottom by a truncated cone, to reproduce a stranding scenario, and the lateral collision of a double hull with a bridge pier [12]. In this work, the failure parameter $D$ was estimated in 0.993703 .

$D=\int_{0}^{\bar{\varepsilon}_{f}} f\left(\frac{\sigma_{h}}{\bar{\sigma}}\right)_{R T C L} d \bar{\varepsilon}$

$f\left(\frac{\sigma_{h}}{\bar{\sigma}}\right)_{R T C L}=\left\{\begin{array}{cc}0 & \frac{\sigma_{h}}{\bar{\sigma}} \leq-\frac{1}{3} \\ \frac{2+2 \frac{\sigma_{h}}{\bar{\sigma}} \sqrt{12-27\left(\frac{\sigma_{h}}{\bar{\sigma}}\right)^{2}}}{3 \frac{\sigma_{h}}{\bar{\sigma}}+\sqrt{12-27\left(\frac{\sigma_{h}}{\bar{\sigma}}\right)^{2}}} & -\frac{1}{3}<\frac{\sigma_{h}}{\bar{\sigma}}<\frac{1}{3} \\ 0.6065 e^{\left(\frac{3 \sigma_{h}}{2 \bar{\sigma}}\right)} & \frac{\sigma_{h}}{\bar{\sigma}} \geq \frac{1}{3}\end{array}\right.$

The BWH instability criterion [16] combines the shear stress criterion of Bressan and Williams [17] with the local necking analysis in plates presented by Hill [18] so giving a simplified way to determine the onset of local necking in plates by limiting the major principal stress value, equation (6). For this reason, the BWH instability criterion can be only applied to structures subjected to membrane stresses. This formulation was based on the parameters obtained from the power law material model ( $K=646 \mathrm{MPa}, n=0.36$ and $\left.\varepsilon_{1 c}=0.7294\right)$ and the $\beta$ parameter which is defined as the relation between minor and major strain rates in principal directions.

$\sigma_{1}=\left\{\begin{array}{cl}\frac{2 K}{\sqrt{3}}\left(\frac{1+\frac{\beta}{2}}{\sqrt{\beta^{2}+\beta+1}}\right)\left(\frac{2 \varepsilon_{1 c}}{\sqrt{3}} \frac{\sqrt{\beta^{2}+\beta+1}}{(1+\beta)}\right)^{n} & \beta \leq 0 \\ \frac{2 K}{\sqrt{3}} \frac{\left(\frac{2 n}{\sqrt{3}}\right)^{n}}{\sqrt{1-\left(\frac{\beta}{2+\beta}\right)^{2}}} & \beta>0\end{array}\right.$ 


\section{Impact tests in reduced scale naval structures}

\subsection{Drop weight test on $\mathrm{T}$ section beam}

The first naval structure tested in this research was a $\mathrm{T}$ cross-section beam clamped at both ends and collided (or penetrated) in its mid span by a mass with a round headed indenter. The beams were made of mild steel, cut by laser from a $0.25 \mathrm{~mm}$ thickness plate and joined (the reinforcement plate under the horizontal strip) using laser welding. The length of the beam was $50 \mathrm{~mm}$ and the dimensions of its $\mathrm{T}$ cross-section were $12.5 \mathrm{~mm}$ for the strip width and $5.0 \mathrm{~mm}$ for the reinforcement height. The welding process generated a homogeneous and clean fillet weld of about $0.3 \mathrm{~mm}$ size. The T-beams was submitted to impact test of mid span load, figure 4 .

The mass was dropped in free fall over the mid span of the $\mathrm{T}$ cross-section beam and conducted vertically by the linear guide rail system mounted on the low-energy drop weight tester machine. The mass velocity was measured by a Polytec laser Doppler vibrometer, model OFV-323, mounted in the top of the drop weight tester machine. A mass of $0.234 \mathrm{~kg}$ was drop so resulting a velocity of $3.0106 \mathrm{~m} / \mathrm{s}$. The displacement and the acceleration of the indenter were obtained by integrating and deriving the velocity data registered by the vibrometer, respectively. The force was calculated by multiplying the acceleration data by the mass.

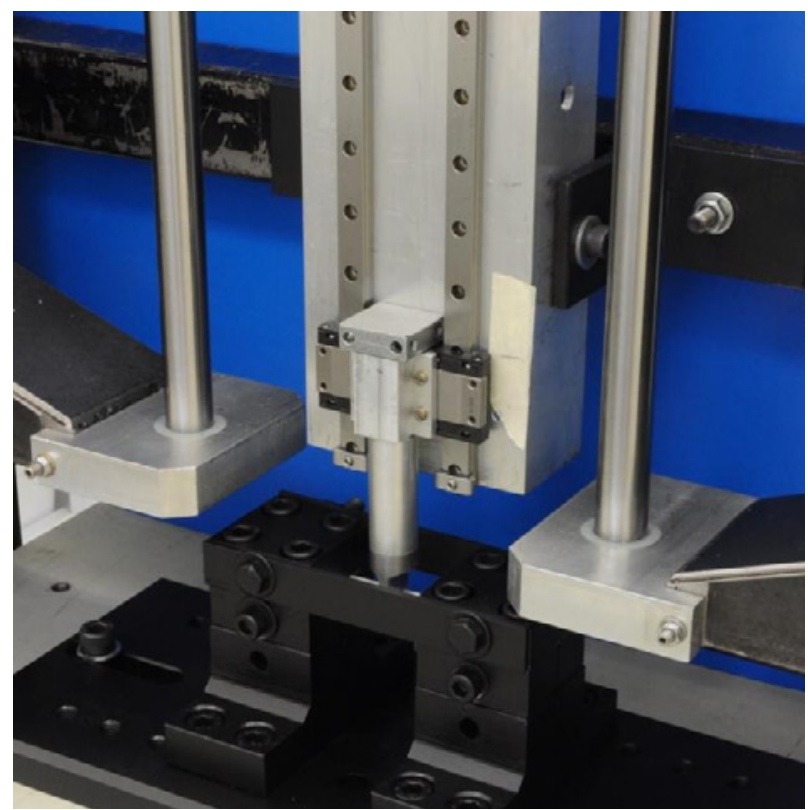

Fig. 4. Set up of the drop weight test of the T-beam.

The drop weight test performed on the T cross-section beam was modelled numerically using the finite element method (FEM). The numerical model was created in the Ansys R13 code, the LS-dyna 971 was used as numerical explicit solver and the LS-Pre-Post 4.0 for the postprocessing of results. All the setup geometry for the drop weight test of the beam was simplified in the numerical model as a beam full constrained in both ends and being collided in mid-span by a solid indenter.

The $\mathrm{T}$ cross-section beam geometry was modelled using regular square $0.5 \times 0.5 \mathrm{~mm}$ shell elements and considering the mid-plane dimensions of the beam so giving a total of 3700 shell elements, figure 5 .

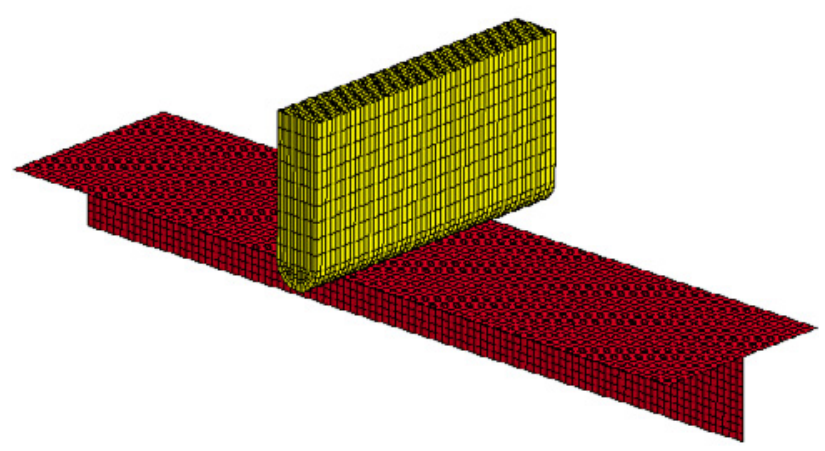

Fig. 5. Numerical model of the T-beam impact test.

The Voce model was used to describe the elastic plastic behaviour of the material and the CowperSymonds formulation was used to include the dynamic sensitivity to high strain rates using the material parameters estimated formerly. No failure criterion was included in the material model since experimental tests of the $\mathrm{T}$ beam showed no material failure.

The indenter has a circular format with $3.0 \mathrm{~mm}$ diameter, its geometry was modelled using $0.5 \times 0.5 \mathrm{~mm}$ hexahedral solid elements with linear elastic material model and the standard properties of the steel (elasticity modulus of $205 \mathrm{GPa}$ and Poisson coefficient of 0.3 ) giving a total of 13500 solid elements.

The structural response, defined by the force displacement curve of the indenter, for the experimental and numerical tests were compared in figure 6 . FE model presented a progressive increasing of the response force similar to the experiment. However, a notable difference in the response force took place at the beginning of the contact which mitigate as the indenter penetration progressed.

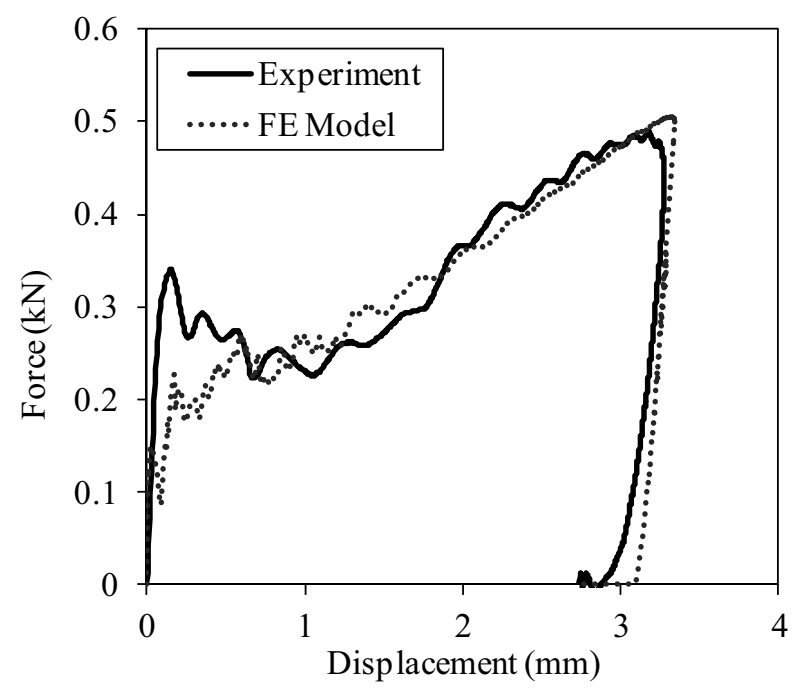

Fig. 6. Structural response of impact test in T-beam. 


\subsection{Impact test on double plate panel}

The second naval structure analyzed in this research was the penetration damage by central impact in a double plate panel. The structure was also constructed using mild steel plate of $0.25 \mathrm{~mm}$ thickness. The panel has a $77.3 \times 77.3 \mathrm{~mm}$ square shape, $21.8 \mathrm{~mm}$ height and includes an internal cross reinforcement, figure 7 . The panel parts were manufactured by laser cutting and the panel parts were conceived to be mounted using tight housing slots to ensure accurate dimensions in the assembly. Once assembled, all parts were joined by laser welding taken into account a progressive and alternate welding sequence to avoid geometry distortions induced by the welding heating.

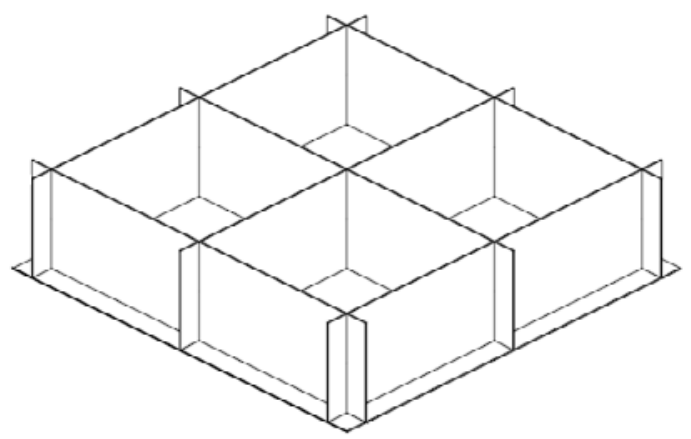

Fig. 7. Inner cross reinforcement of the double plate panel.

This naval structure is far significantly more complex than the T-beam structure. The inner cross reinforcement presents diverse combined collapse modes as progressive buckling and tearing [19]. On the other side, the upper plate is subjected to a general membrane stretching effect and a depth penetration in the contact region.

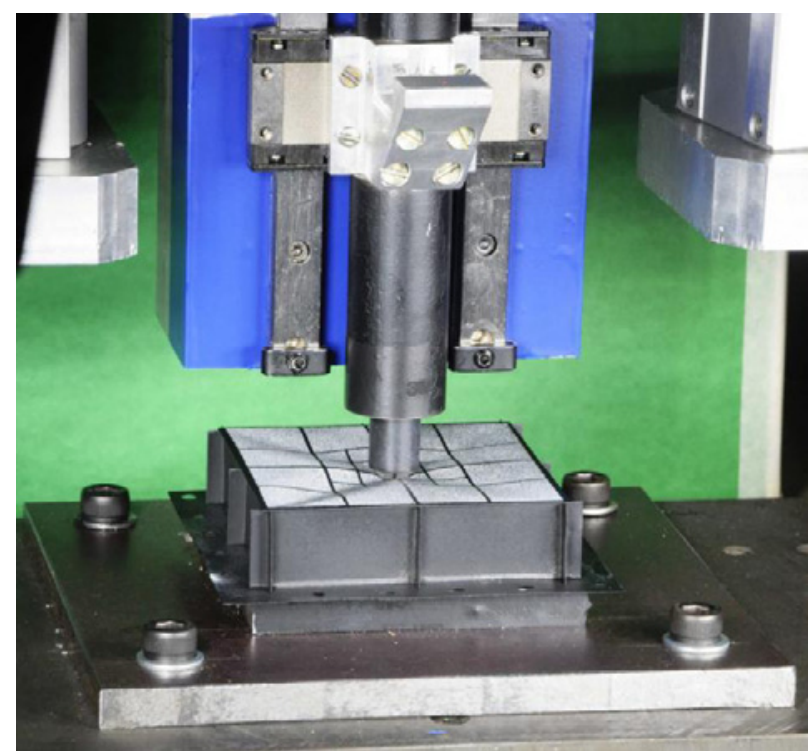

Fig. 8. Impact test performed in double plate panel.

The mass was also dropped in free fall over the middle area of the plate and conducted in the vertical direction by the linear guide rail system of the lowenergy drop weight tester machine, figure 8 . The impacting mass was fixed in $0.588 \mathrm{~kg}$ and accelerated by an external device to achieve an initial velocity of 7.065 $\mathrm{m} / \mathrm{s}$. The mass indenter geometry is cylindrical shape $(\varnothing 12.5 \mathrm{~mm})$ with a $90^{\circ}$ cone head spherically smoothed with $3.4 \mathrm{~mm}$ radius. The panel laid freely in a metallic square holed base of $83.8 \times 83.8 \mathrm{~mm}$ external and $63.8 \times 63.8 \mathrm{~mm}$ internal dimensions.

Similarly to the $\mathrm{T}$ cross-section beam test, the acquisition of the force and displacement of the mass during the impact was done using the Polytec laser Doppler vibrometer, model OFV-323, mounted in the top of the drop weight tester machine.

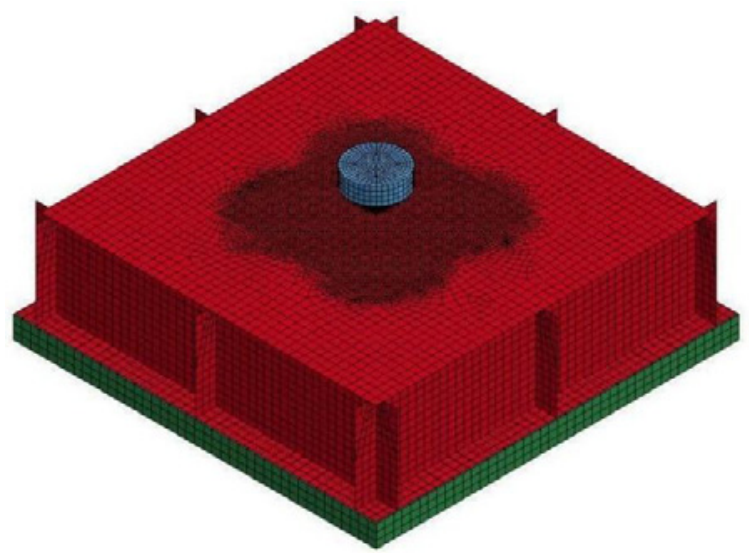

Fig. 9. Numerical model of the cross panel impact test.

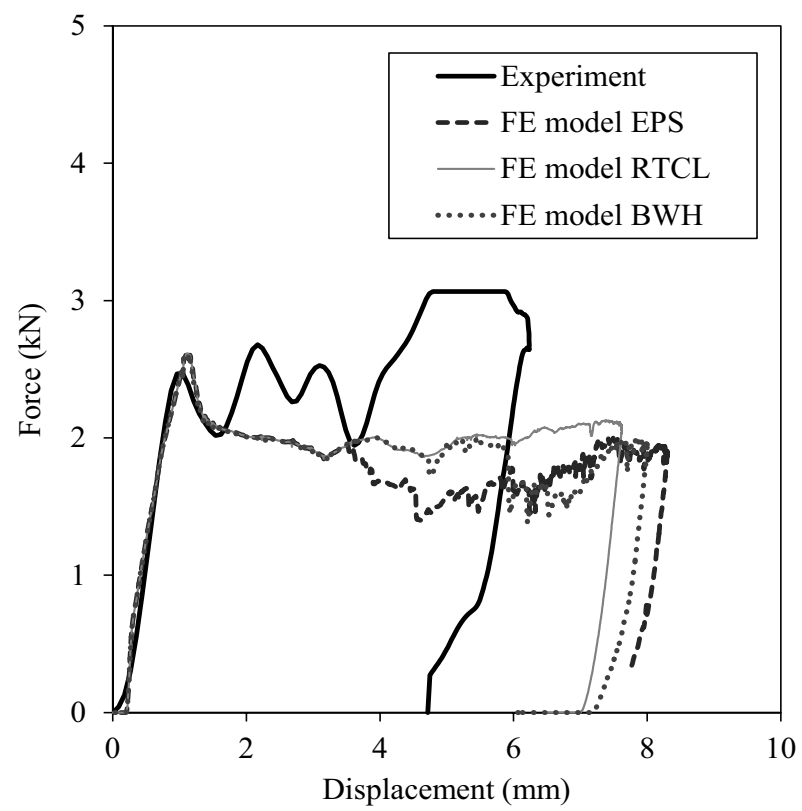

Fig. 10. Structural response of impact test in double plate panel.

Similarly to the numerical model of the $\mathrm{T}$ crosssection beams, the double plate panel model was created in the Ansys R13 code, the LS-dyna 971 was used as numerical solver and the LS-Pre-Post 4.0 for the postprocessing of results.

The panel was modelled using regular square $0.5 \times 0.5$ $\mathrm{mm}$ shell elements in the contact areas and $2.0 \times 2.0 \mathrm{~mm}$ on the remainder areas so resulting in a total of 16796 shell elements for the panel, figure 9. Both the indenter and the square base were modelled using $2.0 \times 2.0 \times 2.0$ 
$\mathrm{mm}$ hexahedral solid elements with linear elastic material model and the standard properties of the steel so resulting in a total of 5220 solid elements. The entire geometry of the panel was conceived considering the actual mid-plane dimensions of the specimen. The structural response, also defined by the force displacement curve of the indenter, for the experimental and numerical tests are shown in figure 10 .

Finite element model responses showed a similar peak force in the beginning of the contact between the indenter and the double plate panel but then this agreement stops after this peak when the progressive buckling of the cross reinforcement of the double plate panel takes place. The finite element models with the RTCL failure criterion showed no structural failure and the models with the EPS and BWH failure criteria showed similar collapse of elements due to membrane stretching and progressive buckling.

\section{Conclusions}

The optimization procedure to evaluate the stress strain curve in quasi static conditions was performed. This evaluation resulted in a more precise mechanical characterization of the material given the good agreement of experimental test with the numerical modelling responses of the uniaxial tensile test.

Similarly, the agreement of the force displacement responses of the experimental test and numerical modelling of the impact test in the $\mathrm{T}$ cross-section beam demonstrated also a good precision of the mechanical characterization of the material in dynamic conditions.

On the other hand, the double plate panel with cross reinforcement is significantly more complex than the beam structure. Superposition of diverse combined collapse modes as progressive buckling, tearing, penetration and membrane stretching were observed so making difficult the evaluation of the structural response and the collapse modes. For a more precise analysis, other structural tests with intermediate complexity were required to clarify the phenomena around this kind of event.

Moreover, given that the reinforced panel is subjected to higher straining velocities than the $\mathrm{T}$ beam, it is also important to point out that the stresses increment due to dynamic conditions affects significantly the structural response of the panel, particularly related to the membrane stretching of the upper plate as stated in theoretical approaches [5]. So a more meticulous analysis of the dynamic effect on the stress-strain curves need to be made to model better events at higher strain rates, as the impact test of the double plate panel.

\section{Acknowledgements}

This work was financially supported by FAPESP (Sao Paulo State Foundation for Research Support, process $\mathrm{n}^{\circ}$ 2011/11733-3) and FINEP (Projects and Studies Finance).

\section{References}

1. M.A.G. Calle, M. Alves, Int. C. Mech. Eng. (2011).

2. R.E. Oshiro, M.A.G. Calle, L.M. Mazzariol, M. Alves, Int. C. Mech. Eng. (2011)

3. K. Hagiwara, H. Takanabe, H. Kawano, Int. J. Imp. Eng., 1, 3 (1983)

4. L.M. Mazzariol, M.A.G. Calle, R.E. Oshiro, M. Alves, Mec. Comp. 29 (2010)

5. L.M. Mazzariol, Master thesis EPUSP (2012)

6. M.A.G. Calle, M. Alves, Int. Sym. Sol. Mech. (2013)

7. LSTC, LS-Dyna manual (2007)

8. N. Jones, Structural Impact (1997)

9. G. Wang, J. Spencer, Y. Chen, Mar. Str., 15 (2002)

10. K. Wisniewski, P. Kolakowski, Fin. Elem. Anal. Des., 39 (2003)

11. D.P. Servis, M. Samuelides, NTUA report (1999)

12. R. Törnqvist, PhD thesis DTU (2003)

13. J. Rice, D. Tracey, J. Mechs. Phys. Sols. 17 (1969)

14. M.G. Cockcroft, D.J. Latham, J.Inst.Met., 96 (1968)

15. T. Wierzbicki, H. Werner, MIT report (1998)

16. H.S. Alsos, PhD thesis NTNU (2008)

17. J.D. Bressan, J.A. Williams, Int. J. Mech. Sci. 25 (1983)

18. R. Hill, J. Mechs. Phys. Sols., 1 (1952)

19. S. Zhang, PhD thesis DTU (1999) 